Research Article

\title{
On the Topological Dynamics of Dynamical Manifolds and Their Fundamental Group
}

\author{
M. Abu-Saleem (i) and Omar Almallah \\ Department of Mathematics, Al-Balqa Applied University, Salt, Jordan \\ Correspondence should be addressed to M. Abu-Saleem; mohammedabusaleem2005@yahoo.com
}

Received 15 May 2021; Accepted 22 July 2021; Published 14 August 2021

Academic Editor: Xiangfeng Yang

Copyright (c) 2021 M. Abu-Saleem and Omar Almallah. This is an open access article distributed under the Creative Commons Attribution License, which permits unrestricted use, distribution, and reproduction in any medium, provided the original work is properly cited.

\begin{abstract}
The paper aims to deduce the relation between the category of topology and algebra from viewpoint of geometry and dynamical system. We introduce and define a dynamical manifold as a manifold associated with a time parameter. We obtain the induced chain of topological dynamics on the fundamental group from the chain of dynamical maps on a dynamical manifold. For many adjunctions in this context, we deduce the limit topological dynamics and conditional topological dynamics on the fundamental group. We use the category of commutative diagrams as chains of dynamical manifolds to deduce the chains on fundamental groups. Also, we describe how the manifold changes in a dynamical system from the view of the fundamental group.
\end{abstract}

\section{Introduction and Definitions}

A dynamical system might be an arrangement that grows in time via the iterative application of a dynamical rule. The evolution rule shows the transformation of the real state under its conditions as well as possibly earlier conditions. The fact that state evolutions must be based on the system's states suggests that the dynamics are recursive. A dynamical system, in particular, is not a simple input-output transformation; rather, the real states are determined by the system's history. Input does not need to be given to the system continuously, and it may be sufficient if the input is only given as an initial state, and the system is then allowed to evolve according to its internal dynamical rule, and this will characterize the normal model of the dynamical system. The transition rules for the dynamical system are usually determined by a set of parameters. Exploring the qualitative nature of this dependency is an important aspect of dynamical systems theory $[1,2]$.

A dynamical system with components, a state space $D$, a time set $T \subseteq R$, being an additive semigroup, and an operator of evolution $\Psi: D \times T \longrightarrow D$ satisfies the group property, that is, $\Psi(y, 0)=y$ and $\Psi\left(\Psi\left(y, t_{1}\right), t_{2}\right)=$ $\Psi\left(y, t_{1}+t_{2}\right), \forall y \in D$ and $t_{1}$ and $t_{2} \in D$ [3]. If whenever each point of $N$ has a neighborhood that is homeomorphic to an open which contained in $R^{n}$, then we say a topological space $N$ is locally Euclidean of dimension $n$. However, a second countable Hausdorff space which is locally Euclidean of dimension $n$ is called an $n$-dimensional topological manifold. Indeed, the only topological manifolds were considered in this paper, for the most part, call them $n$-dimensional manifolds or manifolds. A submanifold of a manifold $N$ is a subset $L$ which itself has the structure of a manifold [4]. A surface (or 2-manifold) is a space that is homeomorphic to a nonempty subset of a finite-dimensional Euclidean space and in which every point has a neighborhood homeomorphic to $R^{2}[5,6]$. Given a topological space $X$ with a subspace $B \subseteq X$, we say $B$ is a retract of $X$ if there is a continuous map $r: X \longrightarrow B$ for which $r(b)=b, \forall b \in B[5,7]$. Given spaces $X_{1}$ and $X_{2}$ with $a_{0} \in X_{1}, b_{0} \in X_{2}$, and $X_{1} \cap X_{2}=\phi$, then the wedge sum $X_{1} \vee X_{2}$ is the quotient of $X_{1} \cup X_{2}$ under the identification $a_{0} \sim b_{0}[8,9]$. Given a closed connected twodimensional manifold $\Sigma$ and $n \in N_{0}$, we define $\Sigma$ minus $n$ open disks as the result of removing the interiors of $n$ disjoint closed disks. The result is a compact, connected 2-dimensional manifold with $n$ boundary components, and we write $\Sigma_{g, n}=$ the surface of genus $g$ minus $n$ open disks where $g$ and $n \in N_{0} \quad[10,11]$. The relations and connections 
between topological branch and algebra of the dynamical system with evidence and indications were discussed in [12-15]. Poincare introduced the fundamental group through the analysis of curves on surfaces; he classified this group by studying an analytic continuation of multivalued function $\psi$ along with a closed path $p$ in a manifold [11]. Given a topological space $X$ with $x_{1} \in X$. Then, the set of all homotopy classes of loops in $\left(X, x_{1}\right)$ with an operation $\left[f_{1}\right]\left[f_{2}\right]=\left[f_{1} \cdot f_{2}\right]$ is called the fundamental group and denoted by $\pi_{1}\left(X, x_{1}\right)[9,16-18]$.

\section{On the Limit Conditional Topological Dynamics of a Dynamical Manifold}

In this section, we start by defining the topological dynamics, dynamical manifold, limit topological dynamics, and limit conditional topological dynamics on a dynamical manifold, and we obtain the induced limit topological dynamics as a functor from the category of a dynamical manifold into a category of a fundamental group.

Definition 1. Let $I=[a, b]$ be the interval of time; then, it can be written as $\left[t_{1}=a, t_{2}, \ldots, t_{m}, \ldots, b\right]$ where $\lim _{m \longrightarrow \infty} t_{m}=b$. For all $t \in[a, b]$ if a manifold $M$ associate with a time $t$, we call it a dynamical manifold and denote it by $(M, t)$. Also, if a map $\eta_{i}$ associated with a time $t$, we call it a dynamical map and denote it by $\left(\eta_{i}, t\right)$. For simply, we denoted it by $\eta_{i}$.

Definition 2. Suppose that $\left(N_{1}, t_{1}\right)$ and $\left(N_{2}, t_{2}\right)$ are two dynamical manifolds. A continuous dynamical map $\eta_{i}:\left(N_{1}, t_{1}\right) \longrightarrow\left(N_{2}, t_{2}\right)$ is called topological dynamics, if for every dynamical path $\beta$ in a dynamical manifold $\left(N_{1}, t_{1}\right)$, the induced path $\eta \circ \beta$ in a dynamical manifold $\left(N_{2}, t_{2}\right)$ is a dynamical path with variation curvature and torsion (if needed).

Definition 3. Let $\eta_{i}:\left(N_{i-1}, t_{i-1}\right) \longrightarrow\left(N_{i}, t_{i}\right): i=$ $1,2, \ldots, m\}$ be a chain of topological dynamics in a dynamical manifolds; then, we define the limit topological dynamics as $\lim _{m \longrightarrow \infty}\left(\eta_{m}\left(N_{m-1}, t_{m-1}\right)\right)=\lim _{m \longrightarrow \infty} \eta_{m}$ $\left(\eta_{m-1}\left(\ldots \eta_{1}\left(N_{0}, t_{0}\right) \ldots\right)\right)$. Also, if the limit topological dynamics reduces the dimensions of a dynamical manifold, we call it the limit conditional topological dynamical and is denoted by $\lim _{m \longrightarrow \infty}\left(\xi_{m}\left(N^{n}, t_{m}^{n}\right)\right)=\left(N^{n-1}, t^{n-1}\right)$ where $\left(N^{n}, t_{m}^{n}\right)$ is an $n$-dimensional dynamical manifold.

Theorem 1. Given a dynamical manifold $\left(N_{0}, t_{0}\right)$. Then, there is a chain of topological dynamics in dynamical manifolds $\quad\left\{\eta_{i}:\left(N_{i-1}, t_{i-1}\right) \longrightarrow\left(N_{i}, t_{i}\right): i=1,2, \ldots, m\right\}$ which induces $\quad\left\{\hat{\eta}_{i}: \pi_{1}\left(N_{i-1}, t_{i-1}\right) \longrightarrow \pi_{1}\left(N_{i}, t_{i}\right): i=\right.$ $1,2, \ldots m\}$ for which $\lim _{m \longrightarrow \infty}\left(\widehat{\eta}_{m}\left(\pi_{1}\left(N_{m-1}, t_{m-1}\right)\right)\right)=$ $\pi_{1}\left(\lim _{m \longrightarrow \infty}\left(\eta_{m}\left(N_{m-1}, t_{m-1}\right)\right)\right)$.

Proof. The chain of topological dynamics $\left\{\eta_{i}:\left(N_{i-1}, t_{i-1}\right)\right.$ $\left.\longrightarrow\left(N_{i}, t_{i}\right): i=1,2, \ldots, m\right\}$ induces $\left\{\widehat{\eta}_{i}: \pi_{1}\left(N_{i-1}, t_{i-1}\right) \longrightarrow\right.$ $\left.\pi_{1}\left(N_{i}, t_{i}\right): i=1,2, \ldots, m\right\}$ for which $\lim _{m \longrightarrow \infty}\left(\widehat{\eta}_{m}\left(\pi_{1}\right.\right.$ $\left.\left.\left(N_{m-1}, t_{m-1}\right)\right)\right)=\left(\lim _{m \rightarrow \infty}\left(\eta_{m}\left[\left(\alpha, t_{m-1}\right)\right]:\right.\right.$ where $\left(\alpha, t_{m-1}\right)$ is a dynamical loop based at $\left.\left.\left(x_{0}, \quad t_{m-1}\right) \in\left(N_{m-1}, t_{m-1}\right)\right)\right)$ $=\left(\lim _{m \longrightarrow \infty}\left(\left[\eta_{m}\left(\alpha, t_{m-1}\right)\right]: \quad \quad\right.\right.$ where $\eta_{m}\left(\alpha, t_{m-1}\right)$ is a composite loop based at $\left.\left.\eta_{m}\left(x_{0}, t_{m-1}\right) \in \eta_{m}\left(N_{m-1}, t_{m-1}\right)\right)\right)$ $=\left(\left[\lim _{m \longrightarrow \infty}\left(\eta_{m}\left(\alpha, t_{m-1}\right)\right)\right]:\right.$ where $\lim _{m \rightarrow \infty} \eta_{m}\left(\alpha, t_{m-1}\right)$ is a dynamical loop based at $\lim _{m \rightarrow \infty} \quad \eta_{m}\left(x_{0}, t_{m-1}\right) \in$ $\lim _{m \longrightarrow \infty} \eta_{m}\left(N_{m-1}, t_{m-1}\right)=\lim _{m \longrightarrow \infty} \eta_{m} \quad\left(\eta_{m-1}\left(\ldots \eta_{1}\left(N_{0}\right.\right.\right.$, $\left.\left.\left.\left.t_{0}\right) \ldots\right)\right)\right)=\pi_{1}\left(\lim _{m \longrightarrow \infty}\left(\eta_{m}\left(N_{m-1}, t_{m-1}\right)\right)\right)$.

Theorem 2. Let $\left(C_{0}, t_{0}\right)$ be a compact connected dynamical 1-dimensional manifold without boundary. Then,

(i) The chain of conditional topological dynamics $\left\{\xi_{i}:\left(C_{i-1}, t_{i-1}\right) \longrightarrow\left(C_{i}, t_{i}\right): i=1,2, \ldots, m\right\}$ induces $a$ chain of conditional topological dynamics $\left\{\widehat{\xi}_{i}: \pi_{1}\left(C_{i-1}, t_{i-1}\right) \longrightarrow \pi_{1}\left(C_{i}, t_{i}\right): i=1,2, \ldots, m\right\}$ for which $\lim _{m \longrightarrow \infty}\left(\widehat{\xi}_{m}\left(\pi_{1}\left(C_{m-1}, t_{m-1}\right)\right)\right)=0$

(ii) The chain of topological dynamics $\left\{\eta_{i}:\left(C_{i-1}, t_{i-1}\right) \longrightarrow\left(C_{i}, t_{i}\right): i=1,2, \ldots, m\right\}$ induces a chain of topological dynamics $\left\{\widehat{\eta}_{i}: \pi_{1}\left(C_{i-1}, t_{i-1}\right) \longrightarrow \pi_{1}\left(C_{i}, t_{i}\right): i=1,2, \ldots, m\right\}$ for which $\lim _{m \longrightarrow \infty}\left(\widehat{\eta}_{m}\left(\pi_{1}\left(C_{m-1}, t_{i m-1}\right)\right)\right)=0$

Proof

(i) We know that $\left(C_{0}, t_{0}\right)$, the compact connected dynamical 1-dimensional manifold without boundary, is homeomorphic to $\left(S^{1}, t_{0}\right)$, and so, we obtain the following chain of conditional topological dynamics $\eta_{1}:\left(C_{0}, t_{0}\right) \longrightarrow\left(C_{1}, t_{1}\right), \eta_{2}:\left(C_{1}, t_{1}\right) \longrightarrow\left(C_{2}, t_{2}\right), \ldots$, $\eta_{m}:\left(C_{m-1}, t_{m-1}\right) \longrightarrow\left(C_{m}, t_{m}\right)$ for which $\lim _{m \rightarrow \infty}$ $\left.\eta_{m}\left(C_{m-1}, t_{m-1}\right)\right)$ is a point, as shown in Figure 1 , which induces $\widehat{\eta}_{1}: \pi_{1}\left(C_{0}, t_{0}\right) \longrightarrow \pi_{1}\left(C_{1}, t_{1}\right), \widehat{\eta}_{2}: \pi_{1}$ $\left(C_{1}, t_{1}\right) \longrightarrow \pi_{1}\left(C_{2}, t_{2}\right), \ldots, \hat{\eta}_{m}: \pi_{1}\left(C_{m-1}, t_{m-1}\right) \longrightarrow$ $\pi_{1}\left(C_{m}, t_{m}\right)$ for which $\lim _{m \longrightarrow \infty}\left(\widehat{\eta}_{m}\left(\pi_{1}\left(C_{m-1}\right.\right.\right.$ $\left.\left.t_{m-1}\right)\right)=\pi_{1} \quad\left(\lim _{m \longrightarrow \infty} \eta_{m}\left(C_{m-1} t_{m-1}\right)\right)=\pi_{1}(0-$ dimensional dynamical manifold). Hence, $\lim _{m \rightarrow \infty}$ $\left(\widehat{\xi}\left(\pi_{1}\left(C_{m-1}, t_{m-1}\right)\right)=0\right.$.

(ii) Consider the chain of topological dynamics $\xi_{1}:\left(B_{0}\right.$, $\left.t_{0}\right) \longrightarrow\left(B_{1}, t_{1}\right), \xi_{2}:\left(B_{1}, t_{1}\right) \longrightarrow\left(B_{2}, t_{2}\right), \ldots, \xi_{m}:\left(B_{m-1}\right.$, $\left.t_{m-1}\right) \longrightarrow\left(B_{m}, t_{m}\right)$, for which $\lim _{m \longrightarrow \infty}\left(\xi_{m}\left(B_{m-1}\right.\right.$, $\left.\left.t_{m-1}\right)\right)=R^{1}$, as shown in Figure 2, which induces $\widehat{\xi}_{1}: \pi_{1}\left(B_{0}, t_{0}\right) \longrightarrow \pi_{1}\left(B_{1}, t_{1}\right), \widehat{\xi}_{2}: \pi_{1}\left(B_{1}, t_{1}\right) \longrightarrow \pi_{1}$ $\left(B_{2}, t_{2}\right), \ldots, \widehat{\xi}_{m}: \pi_{1}\left(B_{m-1}, t_{m-1}\right) \longrightarrow \pi_{1}\left(B_{m}, t_{m}\right)$, in which $\lim _{m \longrightarrow \infty}\left(\widehat{\xi}\left(\pi_{1}\left(B_{m-1}, t_{m-1}\right)\right)\right)=\pi_{1} \quad\left(\lim _{m \rightarrow \infty}\right.$ $\left.\left(\xi_{m}\left(B_{m-1}, t_{m-1}\right)\right)\right)=\pi_{1}\left(R^{1}\right)$.

Hence, $\lim _{m \rightarrow \infty}\left(\widehat{\xi}\left(\pi_{1}\left(B_{m-1}, t_{m-1}\right)\right)\right)=0$.

Theorem 3. Given the $i$-surface $\Sigma_{0, n}^{i}, 1 \leq n \leq 2$. Then, the two chains of topological dynamics $\left\{\eta_{i}^{j}:\left(\sum_{0, n}^{i-1}, t_{i-1}^{j}\right) \longrightarrow\left(\sum_{0, n}^{i}, t_{i}^{j}\right): i=1,2, \ldots, m, j=1,2\right\} \quad$ induce two chains of topological dynamics $\left\{\widehat{\eta}_{i}^{j}: \pi_{1}\left(\Sigma_{0, n}^{i-1}, t_{i-1}^{j}\right) \longrightarrow \pi_{1}\left(\Sigma_{0, n}^{i} t_{i}^{j}\right): i=1,2, \ldots, m, j=1,2\right\}$ for which rank $\lim _{m \longrightarrow \infty}\left(\hat{\eta}_{i}^{j}\left(\pi_{1}\left(\sum_{0, n}^{m-1}, t_{m-1}^{j}\right)\right)\right)$ is a free abelian group of rank two or identity group. 

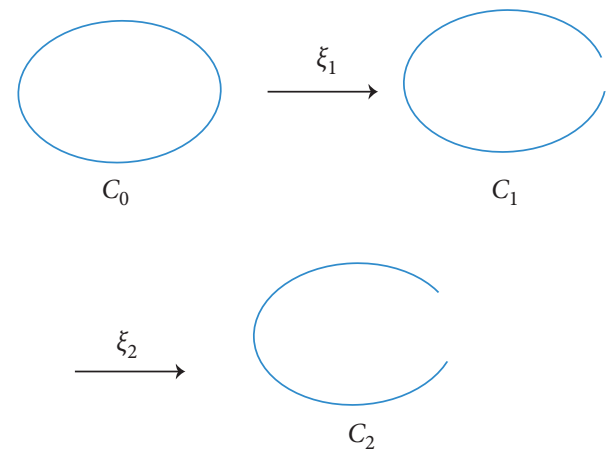

$$
\stackrel{\lim _{m \rightarrow \infty} \xi_{m}}{\longrightarrow} \cdots \text { (Point) }
$$

FIgURE 1: The chain of conditional topological dynamics on 1dimensional manifold.

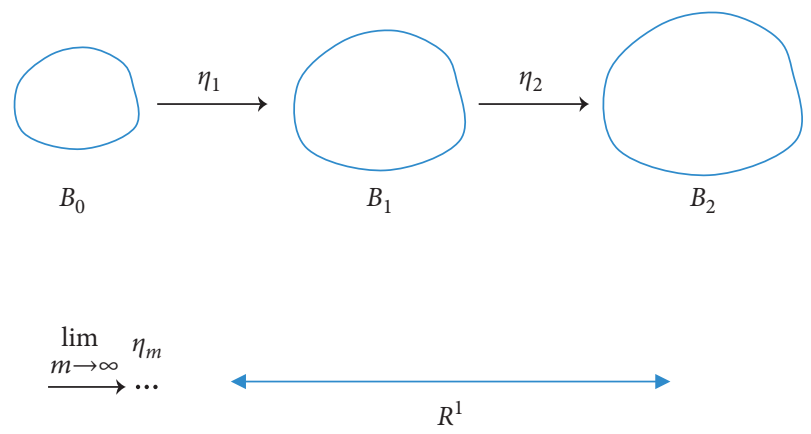

FIgURE 2: The chain of topological dynamics on 1-dimensional manifold.

Proof. First, $\quad$ let $\quad \eta_{1}^{1}:\left(\Sigma_{0, n}^{0}, t_{1}^{1}\right) \longrightarrow\left(\Sigma_{0, n}^{1}, t_{1}^{1}\right), \eta_{2}^{1}$ : $\left(\Sigma_{0, n}^{1}, t_{2}^{1}\right) \longrightarrow\left(\Sigma_{0, n}^{2}, t_{2}^{1}\right), \ldots, \eta_{m}^{1}:\left(\Sigma_{0, n}^{m-1}, t_{m-1}^{1}\right) \longrightarrow\left(\Sigma_{0, n}^{m}, t_{m}^{1}\right)$ be a chain of topological dynamics for which $\lim _{m \longrightarrow \infty} \eta_{m}^{1}\left(\Sigma_{0, n}^{m-1}, t_{m-1}^{1}\right)=\Sigma_{0,0}$, as shown in Figures 3 and 4 , which induces a chain $\hat{\eta}_{1}^{1}: \pi_{1}\left(\Sigma_{0, n}^{0}, t_{0}^{1}\right) \longrightarrow \pi_{1}\left(\Sigma_{0, n}^{1}, t_{1}^{1}\right)$, $\hat{\eta}_{2}^{1}: \pi_{1}\left(\Sigma_{0, n}^{1}, t_{1}^{1}\right) \longrightarrow \pi_{1}\left(\Sigma_{0, n}^{2}, t_{2}^{1}\right), \ldots, \quad \hat{\eta}_{m}^{1}: \pi_{1}\left(\Sigma_{0, n}^{m-1}, t_{m-1}^{1}\right)$ $\left.\longrightarrow \pi_{1}\left(\Sigma_{0, n}^{m}, t_{m}^{1}\right)\right\}$ such that $\lim _{m \longrightarrow \infty}\left(\widehat{\eta}_{m}^{1}\left(\pi_{1}\left(\Sigma_{0, n}^{m-1}, t_{m-1}^{1}\right)\right)\right)$ $=\pi_{1}\left(\lim _{m \longrightarrow \infty}\left(\eta_{m}^{1}\left(\sum_{0, n}^{m-1}, t_{m-1}^{1}\right)\right)\right)$, and so, $\lim _{m \longrightarrow \infty}\left(\widehat{\eta}_{m}\right.$ $\left.\left(\pi_{1}\left(\Sigma_{0, n}^{m-1}, t_{m-1}^{1}\right)\right)\right)=\pi_{1}\left(\Sigma_{0,0}\right)=0$.

Also, the other chain of topological dynamics $\eta_{1}^{2}:\left(\Sigma_{0, n}^{0}, t_{0}^{2}\right) \longrightarrow\left(\Sigma_{0, n}^{1}, t_{1}^{2}\right), \eta_{2}^{2}:\left(\Sigma_{0, n}^{1}, t_{1}^{2}\right) \longrightarrow\left(\Sigma_{0, n}^{2}, t_{2}^{2}\right), \ldots$, $\eta_{m}^{2}:\left(\sum_{0, n}^{m-1}, t_{m-1}^{2}\right) \longrightarrow\left(\sum_{0, n}^{m}, t_{m}^{2}\right)$ for which $\lim _{m \longrightarrow \infty} \eta_{m}^{2}$ $\left(\left(\sum_{0, n}^{m-1}, t_{m-}^{2}\right)=T^{1}\right.$, as shown in Figure 5, which induces a chain $\widehat{\eta}_{1}^{2}: \pi_{1}\left(\Sigma_{0, n}^{0}, t_{0}^{2}\right) \longrightarrow \pi_{1}\left(\Sigma_{0, n}^{1}, t_{1}^{2}\right), \widehat{\eta}_{2}^{2}: \pi_{1}\left(\Sigma_{0, n}^{1}, t_{1}^{2}\right) \longrightarrow$ $\pi_{1}\left(\Sigma_{0, n}^{2}, t_{2}^{2}\right), \ldots, \quad \hat{\eta}_{m}^{2}: \pi_{1}\left(\sum_{0, n}^{m-1}, t_{m-1}^{2}\right) \longrightarrow \pi_{1}\left(\sum_{0, n}^{m}, t_{m}^{2}\right)$ such that $\quad \lim _{m \longrightarrow \infty}\left(\hat{\eta}_{m}^{2}\left(\pi_{1}\left(\Sigma_{0, n}^{m-1}, t_{m-1}^{2}\right)\right)\right)=\pi_{1}\left(\lim _{m \longrightarrow \infty}\left(\eta_{m}^{2}\right.\right.$ $\left.\left.\left(\Sigma_{0, n}^{m-1}, \quad t_{m}^{2}\right)\right)\right), \quad$ and so, $\pi_{1}\left(\Sigma_{0,1}\right)=\pi_{1}\left(T^{1}\right)$. Hence, $\lim _{m \longrightarrow \infty}\left(\widehat{\eta}_{m}^{2}\left(\pi_{1}\left(\Sigma_{0, n}^{m-1}, t_{m}^{2}\right)\right)\right)$ is a free abelian group of rank 2. Consequently, $\lim _{m \longrightarrow \infty}\left(\hat{\eta}_{m}^{j}\left(\pi_{1}\left(\Sigma_{0, n}^{m-1}, t_{m}^{2}\right)\right)\right)$ is a free abelian group of rank two or identity group.

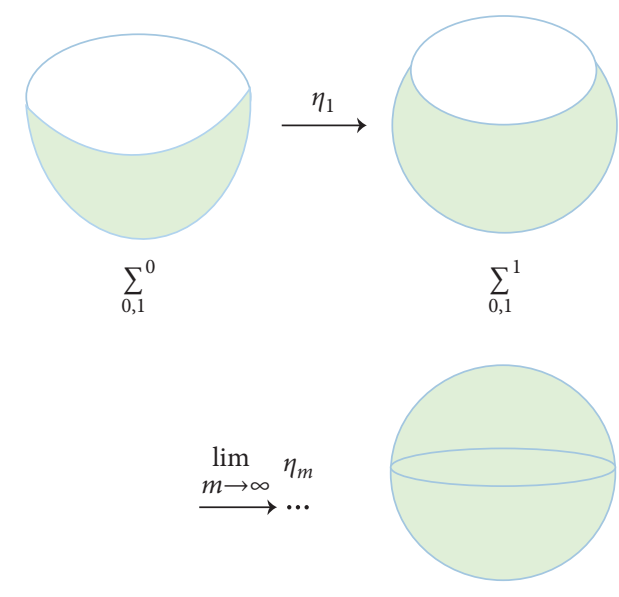

$\sum_{0,0}$

FIgURE 3: Type 1: the chain of topological dynamics on a surface.

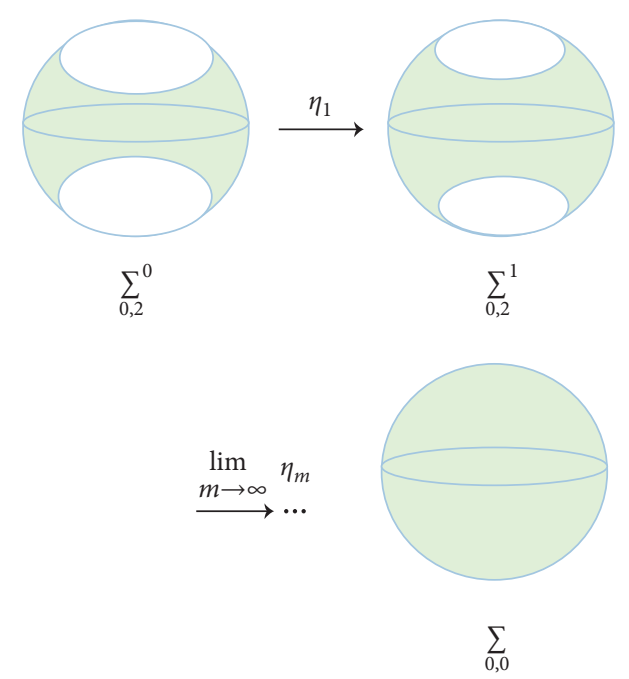

FIgUre 4: Type 2: the chain of topological dynamics on a surface.
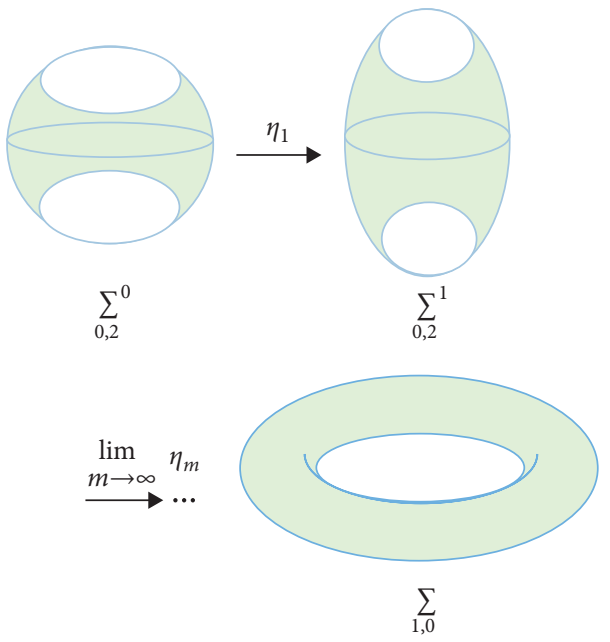

FIgURE 5: Type 3: the chain of topological dynamics on a surface. 


\section{Theorem 4}

(i) Given a compact connected 1-dimensional topological dynamical manifold with boundary $J$ and $N_{0}=J \vee J$. Then, there is a chain of topological dynamics for which rank $\left(\lim _{m \longrightarrow \infty}\left(\widehat{\eta}_{m}\left(\pi_{1}\left(N_{m-1}\right)\right)\right), t_{m-1}\right)=2$

(ii) Let $C_{0}=\{(r, \theta), a \leq r \leq b\}$; then, there is a chain of topological dynamics $\left\{\eta_{i}:\left(C_{i-1}, t_{i-1}\right) \longrightarrow\left(C_{i}, t_{i}\right): i=\right.$ $1,2, \ldots, m\}$, which induces a chain $\left\{\left(\eta_{i}, t_{i}\right): \pi_{1}\left(C_{i-1}\right.\right.$, $\left.\left.\left.\left.t_{i-1}\right)\right) \longrightarrow \pi_{1}\left(C_{i}, t_{i}\right)\right): i=1,2, \ldots, m\right\}$ such that $\lim _{m \rightarrow \infty}\left(\widehat{\eta}_{m}\left(\pi_{1}\left(C_{m-1}, t_{m-1}\right)\right)\right)=0$

\section{Proof}

(i) Let $J$ be a compact connected 1-dimensional topological manifold with boundary; then, $J \approx[a, b]$, and so, $N_{0} \approx[a, b] \vee[a, b]$. Now, consider the following chain of topological dynamics $\eta_{1}:\left(N_{0}, t_{0}\right) \longrightarrow$ $\left(N_{1}, t_{1}\right), \eta_{2}:\left(N_{1}, t_{1}\right) \longrightarrow\left(N_{2}, t_{2}\right), \ldots, \eta_{m}:\left(N_{m-1}\right.$, $\left.\left.t_{m-1}\right) \longrightarrow\left(N_{m}, t_{m}\right)\right\}$, for which $\lim _{m \longrightarrow \infty} \eta_{1}\left(N_{m-1}\right.$, $\left.t_{m-1}\right)=S^{1} \vee S^{1}$, as shown in Figure 6; thus, $\lim _{m \longrightarrow \infty}\left(\widehat{\eta}_{m}\left(\pi_{1}\left(N_{m-1}, t_{m-1}\right)\right)\right)=\pi_{1}\left(\lim _{m \longrightarrow \infty}\left(\widehat{\eta}_{m}\right.\right.$ $\left.\left.\left(N_{m-1}, \quad t_{m-1}\right)\right)\right)=Z * Z$. Consequently, rank $\left(\lim _{m \longrightarrow \infty}\left(\widehat{\eta}_{m}\left(\pi_{1}\left(N_{m-1}, t_{m-1}\right)\right)\right)\right)=2$.

(ii) Let $A=\{(R, \Theta): a \leq R \leq b\} \quad$ and $C=\{(R, \Theta)$ : $0 \leq R \leq b\}$. Also, consider the following chain of topological dynamics $\eta_{1}:\left(A_{0}, t_{0}\right) \longrightarrow\left(A_{1}, t_{1}\right), \eta_{2}$ : $\left(A_{1}, t_{1}\right) \longrightarrow\left(A_{2}, t_{2}\right), \ldots, \eta_{m}:\left(A_{m-1}, t_{m-1}\right) \longrightarrow$ $\left(A_{m}, t_{m}\right)$, for which $\left.\lim _{m \longrightarrow \infty} \eta_{m}\left(A_{m-1}, t_{m-1}\right)\right)=C$, as shown in Figure 7 , which induces $\hat{\eta}_{1}$ : $\left.\left.\pi_{1}\left(A_{0}, t_{0}\right) \longrightarrow \pi_{1}\left(A_{1}, t_{1}\right)\right), \quad \hat{\eta}_{2}: \quad \pi_{1}\left(A_{1}, t_{1}\right)\right) \longrightarrow$ $\pi_{1}\left(A_{2}, t_{2}\right), \ldots, \widehat{\eta}_{m}: \pi_{1}\left(A_{m-1}, t_{m-1}\right) \longrightarrow \pi_{1}\left(A_{m}, t_{m}\right)$ for which $\lim _{m \longrightarrow \infty}\left(\widehat{\eta}_{1}\left(\pi_{1}\left(A_{m-1}, \quad t_{m-1}\right)\right)\right)=\pi_{1}$ $\left(\lim _{m \rightarrow \infty}\left(\widehat{\eta}_{m}\left(A_{m-1}, t_{m}\right)\right)\right)=\pi_{1}(C)$. Consequently, $\lim _{m \rightarrow \infty}\left(\widehat{\eta}_{m}\left(\pi_{1}\left(A_{m-1}, t_{m-1}\right)\right)\right)=0$.

Theorem 5. If $N_{0}=R \cup\left(\Sigma_{0, n} \times Z\right) / \sim$ where given $m \in Z$, we identify $m \in R$ with $((0,0,-1), m) \in \Sigma_{0,0} \times Z$. Then, $\forall k \in N$, and the topological dynamics $\eta_{k}:\left(N_{k-1}\right.$, $\left.t_{k-1}\right) \longrightarrow\left(N_{k}, t_{k}\right) \quad$ induces $\left.\quad \hat{\eta}_{k}: \pi_{1}\left(N_{k-1}, t_{k-1}\right)\right) \longrightarrow$ $\left.\pi_{1}\left(N_{k}, t_{k}\right)\right)$ for which $\lim _{k \longrightarrow \infty}\left(\eta_{k}\left(\pi_{1}\left(N_{k-1}, t_{k-1}\right)\right)\right)=0$.

Proof. Suppose that $N_{0}=R \cup\left(\Sigma_{0, n} \times Z\right) / \sim$. Also, consider the following chain of topological dynamics $\eta_{1}:\left(N_{0}, t_{0}\right) \longrightarrow\left(N_{1}, t_{1}\right), \eta_{2}:\left(N_{1}, t_{1}\right) \longrightarrow\left(N_{2}, t_{2}\right), \ldots$, $\eta_{k}:\left(N_{k-1}, t_{k-1}\right) \longrightarrow\left(N_{k}, t_{k}\right)$, for which $\lim _{k \longrightarrow \infty} \eta_{k}\left(N_{k-1}\right.$, $\left.t_{k-1}\right)=R \cup\left(\Sigma_{0,0} \times Z\right) / \sim$, as shown in Figure 8, which induces $\widehat{\eta}_{1}: \pi_{1}\left(N_{0}, t_{0}\right) \longrightarrow \pi_{1}\left(N_{1}, t_{1}\right), \widehat{\eta}_{2}:\left(N_{1}, t_{1}\right) \longrightarrow\left(N_{2}\right.$, $\left.t_{2}\right), \ldots, \widehat{\eta}_{k}: \quad \pi_{1}\left(N_{k-1}, t_{k-1}\right) \longrightarrow \pi_{1}\left(N_{k}, t_{k}\right)$, for which $\lim _{k \longrightarrow \infty}\left(\hat{\eta}_{k}\left(\pi_{1}\left(N_{k-1}, t_{k-1}\right)\right)\right)=\pi_{1}\left(\lim _{k \longrightarrow \infty}\left(\eta_{k}\left(N_{k-1}, t_{k}\right)\right)\right)$ $=\pi_{1}\left(R \cup\left(\Sigma_{0,0} \times Z\right) / \sim\right)$. Since $R \cup\left(\sum_{0,0} \times Z\right) / \sim$ and $\vee_{i=1}^{\infty} \sum_{0,0}^{i}$ are of the same homotopy type, it follows that $\lim _{k \rightarrow \infty}\left(\eta_{k}\left(\pi_{1}\left(N_{k-1}\right)\right)=0\right.$.
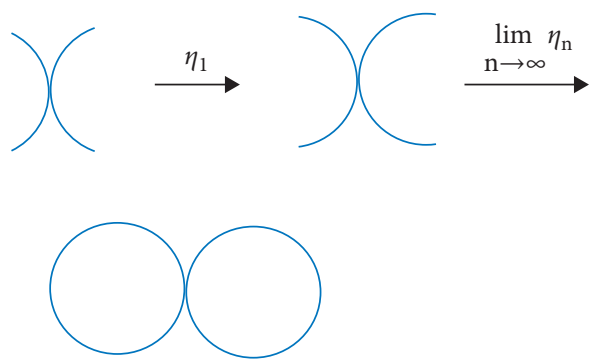

FIgURE 6: The limit topological dynamical on a wedge sum of connected 1-dimensional manifold.
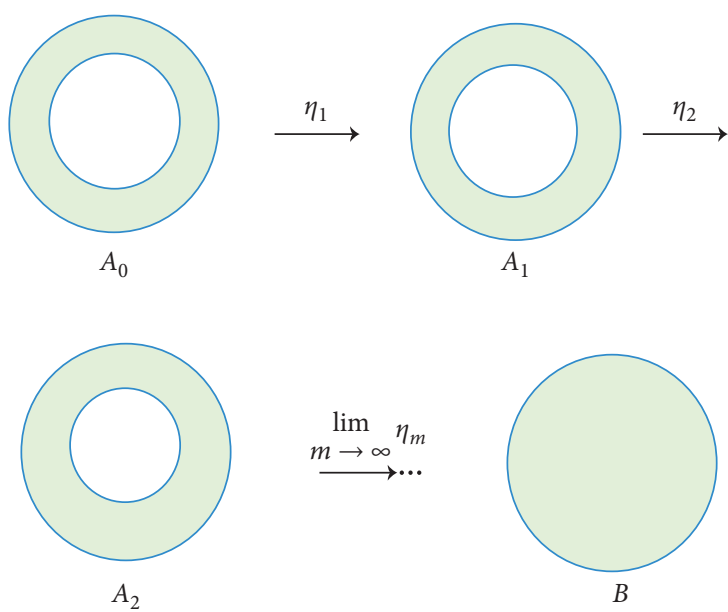

Figure 7: The chain of topological dynamics on an annulus.
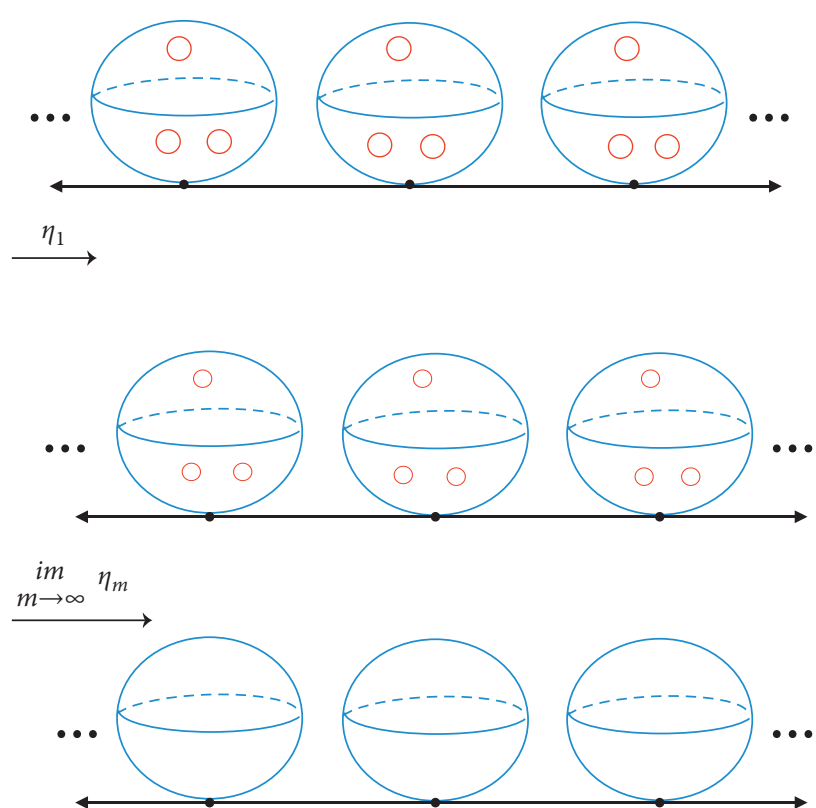

Figure 8: The chain of topological dynamics on $R \cup\left(\Sigma_{0, n} \times Z\right) / \sim$. 
TABLE 1: The chains of commutative diagrams on $\left(N^{n}, t^{n}\right)$ and $\left(L^{n}, t^{n}\right)$.

\begin{tabular}{|c|c|c|c|c|c|c|}
\hline$\left(N^{n}, t^{n}\right)$ & $\stackrel{\xi_{1}^{1}}{\longrightarrow}$ & $\left(N_{1}^{n}, t_{1}^{n}\right)$ & $\stackrel{\xi_{2}^{1}}{\longrightarrow}$ & $\left(N_{2}^{n}, t_{2}^{n}\right)$ & $\stackrel{\lim _{m \rightarrow \infty} \xi_{m}^{1}}{\longrightarrow}$ & $\left(N^{n-1}, t^{n-1}\right)$ \\
\hline$\downarrow r_{1}^{1}$ & & $\downarrow r_{2}^{1}$ & & $\downarrow r_{3}^{1}$ & & $\lim _{m \longrightarrow \infty} \downarrow r_{m}^{1}$ \\
\hline$\left(L^{n}, s^{n}\right)$ & $\stackrel{\xi_{1}^{1}}{\longrightarrow}$ & $\left(L_{1}^{n}, s_{1}^{n}\right)$ & $\stackrel{\xi_{2}^{1}}{\longrightarrow}$ & $\left(L_{2}^{n}, s_{2}^{n}\right)$ & $\stackrel{\lim _{m \rightarrow \infty} \xi_{m}^{1}}{\longrightarrow}$ & $\left(L^{n-1}, s^{n-1}\right)$ \\
\hline$\left(N^{n-1}, t^{n-1}\right)$ & $\stackrel{\xi_{1}^{2}}{\longrightarrow}$ & $\left(N_{1}^{n-1}, t_{1}^{n-1}\right)$ & $\stackrel{\xi_{2}^{2}}{\longrightarrow}$ & $\left(N_{2}^{n-1}, t_{2}^{n-1}\right)$ & $\stackrel{\lim _{m \rightarrow \infty} \xi_{m}^{2}}{\longrightarrow}$ & $\left(N^{n-2}, t^{n-2}\right)$ \\
\hline$\downarrow r_{1}^{2}$ & & $\downarrow r_{2}^{2}$ & & $\downarrow r_{3}^{2}$ & & $\lim _{m \longrightarrow \infty} \downarrow r_{m}^{2}$ \\
\hline$\left(L^{n-1}, s^{n-1}\right)$ & $\stackrel{\xi_{1}^{2}}{\longrightarrow}$ & $\left(L_{1}^{n-1}, s_{1}^{n-1}\right)$ & $\stackrel{\xi_{2}^{2}}{\longrightarrow}$ & $\left(L_{2}^{n-1}, s_{2}^{n-1}\right)$ & $\lim _{m \rightarrow \infty} \xi_{m}^{2}$ & $\left(L^{n-2}, s^{n-2}\right)$ \\
\hline$\vdots$ & $\vdots$ & $\vdots$ & $\vdots$ & $\vdots$ & $\vdots$ & $\vdots$ \\
\hline$\left(N^{1}, t^{1}\right)$ & $\stackrel{\xi_{1}^{n}}{\longrightarrow}$ & $\left(N_{1}^{1}, t_{1}^{1}\right)$ & $\stackrel{\xi_{2}^{n}}{\longrightarrow}$ & $\left(N_{2}^{1}, t_{2}^{1}\right)$ & $\stackrel{\lim _{m \rightarrow \infty} \xi_{m}^{n}}{\longrightarrow}$ & $\left(N^{0}, t^{0}\right)$ \\
\hline$\downarrow r_{1}^{n}$ & & $\downarrow r_{2}^{n}$ & & $\downarrow r_{3}^{n}$ & & $\lim _{m \longrightarrow \infty} \downarrow r_{m}^{n}$ \\
\hline$\left(L^{1}, s^{1}\right)$ & $\stackrel{\xi_{1}^{n}}{\longrightarrow}$ & $\left(L_{1}^{1}, s_{1}^{1}\right)$ & $\stackrel{\xi_{2}^{n}}{\longrightarrow}$ & $\left(L_{2}^{1}, s_{2}^{1}\right)$ & $\stackrel{\lim _{m \rightarrow \infty} \xi_{m}^{n}}{\longrightarrow}$ & $\left(L^{0}, s^{0}\right)$ \\
\hline
\end{tabular}

TABle 2: The induced chains of commutative diagrams.

\begin{tabular}{|c|c|c|c|c|c|c|}
\hline$\pi_{1}\left(N^{n}, t^{n}\right)$ & $\stackrel{\hat{\xi}_{1}^{1}}{\longrightarrow}$ & $\pi_{1}\left(N_{1}^{n}, t_{1}^{n}\right)$ & $\stackrel{\hat{\xi}_{2}^{1}}{\longrightarrow}$ & $\pi_{1}\left(N_{2}^{n}, t_{2}^{n}\right)$ & $\stackrel{\lim _{m} \hat{\xi}_{m}^{1}}{\longrightarrow}$ & $\pi_{1}\left(N^{n-1}, t^{n-1}\right)$ \\
\hline$\downarrow \widehat{r}_{1}^{1}$ & & $\downarrow \widehat{r}_{2}^{1}$ & & $\downarrow \widehat{r}_{3}^{1}$ & & $\lim _{m \longrightarrow \infty} \downarrow \hat{r}_{m}^{1} \pi_{1}$ \\
\hline$\left(L^{n}, s^{n}\right)$ & $\stackrel{\hat{\xi}_{1}^{1}}{\longrightarrow}$ & $\pi_{1}\left(L_{1}^{n}, s_{1}^{n}\right)$ & $\stackrel{\hat{\xi}_{2}^{1}}{\longrightarrow}$ & $\pi_{1}\left(L_{2}^{n}, s_{2}^{n}\right)$ & $\stackrel{\lim _{m} \hat{\xi}_{m}^{1}}{\longrightarrow}$ & $\pi_{1}\left(L^{n-1}, s^{n-1}\right) \pi_{1}$ \\
\hline$\left(N^{n-1}, t^{n-1}\right)$ & $\stackrel{\hat{\xi}_{1}^{2}}{\longrightarrow}$ & $\pi_{1}\left(N_{1}^{n-1}, t_{1}^{n-1}\right)$ & $\stackrel{\hat{\xi}_{2}^{2}}{\longrightarrow}$ & $\pi_{1}\left(N_{2}^{n-1}, t_{2}^{n-1}\right)$ & $\stackrel{\lim _{m} \hat{\xi}_{m}^{2}}{\longrightarrow}$ & $\pi_{1}\left(N^{n-2}, t^{n-2}\right)$ \\
\hline$\downarrow \widehat{r}_{1}^{2}$ & & $\downarrow \widehat{r}_{2}^{2}$ & & $\downarrow \widehat{r}_{3}^{2}$ & & $\lim _{m \longrightarrow \infty} \downarrow \widehat{r}_{m}^{2}$ \\
\hline$\pi_{1}\left(L^{n-1}, s^{n-1}\right)$ & $\stackrel{\hat{\xi}_{1}^{2}}{\longrightarrow}$ & $\pi_{1}\left(L_{1}^{n-1}, s_{1}^{n-1}\right)$ & $\stackrel{\hat{\xi}_{2}^{2}}{\longrightarrow}$ & $\pi_{1}\left(L_{2}^{n-1}, s_{2}^{n-1}\right)$ & $\stackrel{\lim _{m} \hat{\xi}_{m}^{2}}{\longrightarrow}$ & $\pi_{1}\left(L^{n-2}, s^{n-2}\right)$ \\
\hline$\vdots$ & $\vdots$ & $\vdots$ & $\vdots$ & $\vdots$ & $\vdots$ & $\vdots$ \\
\hline$\pi_{1}\left(N^{1}, t^{1}\right)$ & $\stackrel{\hat{\xi}_{1}^{n}}{\longrightarrow}$ & $\pi_{1}\left(N_{1}^{1}, t_{1}^{1}\right)$ & $\stackrel{\hat{\xi}_{2}^{n}}{\longrightarrow}$ & $\pi_{1}\left(N_{2}^{1}, t_{2}^{1}\right)$ & $\stackrel{\lim _{m} \hat{\xi}_{m}^{n}}{\longrightarrow}$ & $\pi_{1}\left(N^{0}, t^{0}\right)$ \\
\hline$\downarrow \widehat{r}_{1}^{n}$ & & $\downarrow \widehat{r}_{2}^{n}$ & & $\downarrow \hat{r}_{3}^{n}$ & & $\lim _{m \longrightarrow \infty} \downarrow \widehat{r}_{m}^{n}$ \\
\hline$\pi_{1}\left(L^{1}, s^{1}\right)$ & $\stackrel{\hat{\xi}_{1}^{n}}{\longrightarrow}$ & $\pi_{1}\left(L_{1}^{1}, s_{1}^{1}\right)$ & $\stackrel{\hat{\xi}_{2}^{n}}{\longrightarrow}$ & $\pi_{1}\left(L_{2}^{1}, s_{2}^{1}\right)$ & $\stackrel{\lim _{m} \hat{\xi}_{m}^{n}}{\longrightarrow}$ & $\pi_{1}\left(L^{0}, s^{0}\right)$ \\
\hline
\end{tabular}

\section{The Limit Conditional Topological Dynamics on an $\boldsymbol{n}$-Dimensional Dynamical Manifold}

We now generalized the results for conditional topological dynamics on an $n$-dimensional dynamical manifold and describe a commutative diagram of induced homomorphism.

Theorem 6. Suppose that $\left(N^{n}, t^{n}\right)$ is an n-dimensional $d y$ namical manifold and $\left(L^{n}, t^{n}\right)$ is a subdynamical manifold of $N^{n}$, and let $\xi_{i}^{j}$ and $r_{i}^{j}$, i and $j=1,2, \ldots, n$, are sequences of conditional topological dynamics and dynamical retractions. Then, the chain of a commutative diagram on dynamical manifolds and subdynamical manifolds induces a chain of a commutative diagram on the fundamental groups.

Proof. Consider the chains of commutative diagrams, as shown in Table 1.

Since the fundamental group is a functor, we get the induced chain of a commutative diagram, as shown in Table 2.

\section{Conclusion}

The relation between the induced limit conditional topological dynamics and induced limit dynamical retractions on the fundamental group is deduced from the viewpoint of 
limit conditional topological dynamics and limit dynamical retractions on dynamical manifolds.

\section{Data Availability}

The data used to support the findings of this study are included within the article.

\section{Conflicts of Interest}

The authors declare that they have no conflicts of interest.

\section{References}

[1] J. Jost, Dynamical Systems, Examples of Complex Behaviour, Springer-Verlag, Berlin, Germany, 2005.

[2] T. Lukashiv, "One form of lyapunov operator for stochastic dynamic system with markov parameters," Journal of Mathematics, vol. 2016, Article ID 1694935, , 2016.

[3] H. Broer and F. Takens, Dynamical Systems and Chaos, Springer-Verlag New York, New York, NY, USA, 2011.

[4] J. M. Lee, Introduction to Topological Manifolds, SpringerVerlag New York, New York, NY, USA, 2010.

[5] W. S. Massey, Algebraic Topology, An Introduction, Harcourt, Brace \& World Inc.,, New York, NY, USA, 1967.

[6] R. Messer and P. Straffin, Topology Now, Classroom Resource Materials, Mathematical Association of America, Washington, DC, USA, 2006.

[7] M. Abu-Saleem, "Retractions and homomorphisms on some operations of graphs," Journal of Mathematics, vol. 2018, pp. 1-4, Article ID 7328065, 2018.

[8] M. Aktay and M. Özdemir, "Some results on generalized patasuzuki type contractive mappings," Journal of Mathematics, vol. 2021, pp. 1-9, Article ID 2975339, 2021.

[9] A. Hatcher, Algebraic Topology, Cambridge University Press, Cambridge, UK, 2002.

[10] J. McCleary, A First Course in Topology, Continuity and Dimension, American Mathematical Society, New York, NY, USA, 2006.

[11] J. Stillwell, Classical Topology and Combinatorial Group Theory, Springer-Verlag, New York Inc, NY, USA, 1993.

[12] M. Abu-Saleem, "The Minimum deformation retract on the wormhole spacetime," International Journal of Geometric Methods in Modern Physics, vol. 17, no. 13, pp. 1-10, 2020.

[13] M. Abu-Saleem, "Dynamical chaotic homotopy group and its applications," International Journal of Nonlinear Science, vol. 11, no. 2, pp. 206-212, 2011.

[14] F. W. Aquino and B. M. Wong, "Additional insights between fermi-löwdin orbital SIC and the localization equation constraints in SIC-DFT," The Journal of Physical Chemistry Letters, vol. 9, no. 22, pp. 6456-6462, 2018.

[15] M. A. Choudhary, “A note on L. C. Q. K. Manifolds," Journal of Dynamical Systems and Geometric Theories, vol. 18, no. 2, pp. 145-161, 2020.

[16] J. Brazas, "The fundamental group as a topological group," Topology and Its Applications, vol. 160, no. 1, pp. 170-188, 2013.

[17] U. Bruzzo and B. Graña Otero, "Higgs varieties and fundamental groups," Journal of Geometry and Physics, vol. 128, pp. 12-19, 2018.

[18] G. Conner and C. Kent, "Fundamental groups of locally connected subsets of the plane," Advances in Mathematics, vol. 347, pp. 384-407, 2019. 